(C) 2016 IEEE. Personal use of this material is permitted. Permission from IEEE must be obtained for all other uses, in any current or future media, including reprinting/republishing this material for advertising or promotional purposes, creating new collective works, for resale or redistribution to servers or lists, or reuse of any copyrighted component of this work in other works. 


\title{
Kinematic Control of an Autonomous Underwater Vehicle- Manipulator System (AUVMS) using Autoregressive Prediction of Vehicle Motion and Model Predictive Control*
}

\author{
Jonathan Woolfrey, Dikai Liu, Marc Carmichael
}

\begin{abstract}
Autonomous Underwater Vehicle-Manipulator Systems (AUVMS) operating in shallow waters or near-surface environments may be exposed to wave disturbances which will cause undesired motion of the end effector. This paper presents a method to maneuver the manipulator joints and counteract undesired motion of the vehicle body, in order to maintain a steady end-effector position in the inertial frame. An Autoregressive (AR) model is used to predict vehicle motion, and then combined with Model Predictive Control (MPC) to optimize joint motion. Simulation was conducted using real data to verify the efficacy of this method.
\end{abstract}

\section{INTRODUCTION}

Autonomous Underwater Vehicle-Manipulator Systems (AUVMS) operating in near-surface environments may be subject to wave disturbances which affect the efficacy of their control. The motion induced on the vehicle body and manipulator will impede the ability of the end effector to maintain a steady pose in the inertial frame required for intervention tasks, such as object handling, surface cleaning, and inspection in the environment. The high frequency and stochastic nature of water flow means reactive control for task error may not be sufficient. If we can predict how waves will affect the motion of the vehicle body, then we ought to be able to maneuver the manipulator joints to counteract this motion.

AUVs have previously been developed for observational tasks in deep sea environments where water currents may be large but predominantly time-invariant. However, there has been increasing development of AUVs with manipulator systems designed to interact with the objects in its surroundings. Notable examples include the GIRONA500 [1], TRIDENT [2], and SAUVIM [3][4][5].

The effects of wave disturbances on control of manipulators on non-inertial platforms have hitherto been largely concerned with robots operating on ships. The main challenge pertains to the nonlinearity and stochastic nature of water currents, making it difficult to predict and counteract. Several methods have been employed to forecast wave motion, such as treating waves as a Gaussian process with

*Research supported by the Australian Research Council (ARC) Linkage Project (LP150100935) and the Roads and Maritime Services of NSW.

All authors are with the Centre for Autonomous Systems (CAS) at the University of Technology Sydney (UTS), CB11.09.300 81 Broadway Ultimo, NSW 2007, Australia. linear relationships between disturbance observations [6], using superposition of sinusoidal waves, and autoregression [7]. Autoregressive (AR) models are such that an observation of $Y_{t}$ is regressed on values of $Y_{t-i}$. This ability to predict future events based on historical data is particularly useful for processes with no identifiable deterministic model that is otherwise regarded as stochastic; i.e. waves.

Model Predictive Control (MPC) is a control method known for its robustness to uncertainty and disturbances. It has been successfully applied to path planning of AUVs while incorporating uncertainty in the future hydrodynamic disturbances [8][9]. MPC optimizes an objective function by considering the total cost of future states and controls at a horizon of $\mathrm{N}$ future steps. Therefore, it is a useful method when knowledge of future conditions is available for consideration in the control plan. However, path-planning of AUVs with MPC operate on the basis of stable and/or previously mapped deep ocean currents. This may be invalid for near-surface operation.

The utility of AR models, coupled with its predictive abilities, make it a suitable choice for integration with MPC. Its diversity is exemplified in disturbance prediction and rejection of feed flow rates in chemical processing [10], climate control within buildings, whilst factoring in weather forecasts [11], and rigid body dynamics by eliminating tremor in microsurgery via teleoperation [12].

Wave disturbance to manipulators on ship platforms has also been modeled with AR [7]. Waves in ocean swell can induce large inertial forces on manipulator joints. Forecasts of waves were used to reduce torque loads on joints, and the cost-to-go function in which the manipulator utilized the ship motion to initiate its own. Heave amplitudes experienced in this scenario were on the order of magnitude of $1 \mathrm{~m}$, at a frequency of approximately $0.25 \mathrm{~Hz}$. Pitch angles were of 0.2 radians in amplitude at roughly $0.5 \mathrm{~Hz}$. Because of this low frequency, forecast intervals of $0.5 \mathrm{~s}$ were sufficient.

Conversely, small AUVMS operating near the water's surface may experience wave disturbances of a much higher frequency, and may be much more erratic. This necessitates more frequent forecasting. This paper applies AR to predict vehicle body motion as a disturbance to the end-effector on a kinematic level (as opposed to dynamics). This in turn will translate to task error that will obstruct accurate and smooth motion required for precision intervention operations. By 
sufficiently predicting the vehicle body motion, model predictive control is then used to move the manipulator joints to eliminate this disturbance and keep the end effector steady in the inertial frame.

\section{II.PROBLEM DESCRIPTION \& FORMULATION}

\section{A. Problem Scenario}

An AUVMS is currently being developed to conduct cleaning and inspection on submerged bridge support columns (piles). The exterior of bridge piles must be inspected regularly for cracks, spalling, and corrosion to make an assessment of its structural integrity. Biofouling such as oysters, mussels, and seaweed which grow on the pile surface must be removed before a detailed inspection can be performed.

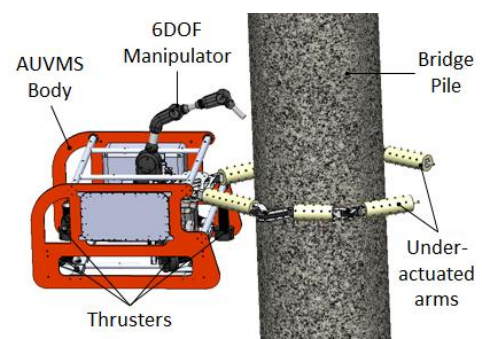

Figure 1. Model of the AUVMS for bridge pile cleaning and inspection.

Cleaning is currently done by human divers using highpressure water. This presents a hazard to human safety, as well as being a physically laborious task. Furthermore, the underwater environment makes it difficult for humans to maneuver, and vision can often be impaired. Automating this process eliminates risks to humans, as well as making the process more productive and efficient.

The density of biofouling is densest in shallow waters up to the intertidal zones, i.e. near the water's surface [13][14]. Effective removal of biofouling requires a steady tool off-set distance and angle of attack relative to the pile surface. Waves in these environments can be erratic and of high frequency, making it difficult for the AUVMS to maintain a steady end-effector pose. Furthermore, water flow patterns vary greatly in direction and magnitude around the circumference of the pile [15]. These may induce large disturbances to the vehicle body. This in turn will affect the ability of the end-effector to maintain an accurate position, and hence impair cleaning operations.

\section{B. Kinematics of the AUVMS}

The Submerged Pile Inspection Robot (SPIR) consists of a holonomic body with 8 thrusters to move in to position in front of a pile. Two under-actuated arms are then used to help secure the vehicle to the pile whilst a third cable-driven, $6 \mathrm{DOF}$ manipulator is to conduct high-pressure water cleaning. It is assumed the arms will maintain proximity to the pile, but ultimately be inadequate at keeping the vehicle rigid.
The position and orientation of the end effector $\boldsymbol{\eta}_{e e}$ with respect to the inertial frame $\{\mathrm{I}\}$ can be expressed through the spatial relationships of the body frame $\{B\}$, the base of the manipulator $\{\mathrm{O}\}$, and the end effector frame $\{\mathrm{E}\}$. Kinematic control can be expressed in terms of vehicle body velocities and the manipulator joint velocities (1). The reader is directed to [4] and [16] for further information on deriving kinematic equations.

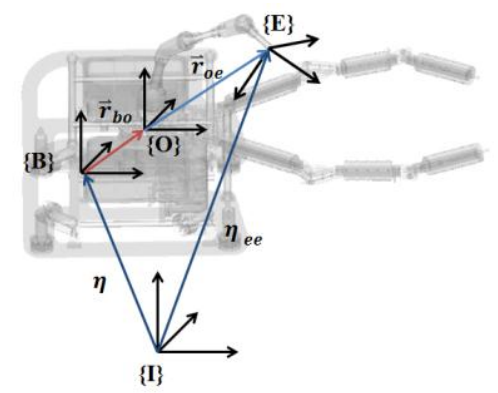

Figure 2. Reference frames on the AUVMS used for kinematics.

The end effector velocity is expressed as:

$$
\dot{\boldsymbol{\eta}}_{e e}=\left[\begin{array}{ll}
\boldsymbol{J}_{e, q}^{-1}\left(\boldsymbol{R}_{I}^{E}\right) J(\boldsymbol{q}) & \boldsymbol{M} \boldsymbol{J}_{e, b}^{-1}\left(\boldsymbol{R}_{I}^{B}\right)
\end{array}\right]\left[\begin{array}{l}
\dot{\boldsymbol{q}} \\
\boldsymbol{v}
\end{array}\right]
$$

Where $\dot{\boldsymbol{q}}$ and $\boldsymbol{v}$ are the manipulator joint velocities and the body velocities respectively. The terms $\boldsymbol{J}_{e, q}^{-1}\left(\boldsymbol{R}_{I}^{E}\right), \boldsymbol{J}_{e, b}^{-1}\left(\boldsymbol{R}_{I}^{B}\right)$ represent the velocity transformation jacobians from the inertial to end-effector frame, and inertial to body frame respectively:

$$
\begin{aligned}
\boldsymbol{J}_{e}^{-1}(\boldsymbol{R}) & =\left[\begin{array}{cc}
\boldsymbol{R} & \mathbf{0}_{3 \times 3} \\
\mathbf{0}_{3 \times 3} & \boldsymbol{J}_{k o}^{-1}\left(\boldsymbol{\eta}_{2}\right)
\end{array}\right] \\
\boldsymbol{J}_{k o}\left(\boldsymbol{\eta}_{2}\right) & =\left[\begin{array}{ccc}
1 & 0 & -s_{\theta} \\
0 & c_{\theta} & c_{\theta} s_{\phi} \\
0 & -s_{\phi} & c_{\theta} c_{\phi}
\end{array}\right]
\end{aligned}
$$

The term $\boldsymbol{J}(\boldsymbol{q})$ is the manipulator Jacobian, and $\theta, \phi$ represent the vehicle pitch and roll respectively. Singular configurations of $\boldsymbol{J}_{k o}\left(\boldsymbol{\eta}_{2}\right)$ are avoided by aligning the body coordinate frame in such a way that $\theta \approx 0$ whilst the vehicle is upright in normal operation. The matrix $\mathbf{M}$ contains displacement vectors between frames, transformed to the inertial frame $\{\mathrm{I}\}$ :

$$
\boldsymbol{M}=\left[\begin{array}{cc}
\boldsymbol{I}_{3} & S\left(-\boldsymbol{R}_{I}^{B} \overrightarrow{\boldsymbol{r}}_{b o}\right)+S\left(-\boldsymbol{R}_{I}^{O} \overrightarrow{\boldsymbol{r}}_{o e}\right) \\
\mathbf{0}_{3 \times 3} & \boldsymbol{I}_{3}
\end{array}\right]
$$

The operator $S(\cdot)$ is a skew symmetric matrix of a $3 \times 1$ vector:

$$
S\left(\left[\begin{array}{lll}
x & y & z
\end{array}\right]^{T}\right)=\left[\begin{array}{ccc}
0 & -z & y \\
z & 0 & -x \\
-y & x & 0
\end{array}\right]
$$




\section{AUTOREGRESSION}

\section{A. Predicting vehicle body motion with AR}

A standard regression is one in which a response variable $Y$ is regressed on a predictor variable $X$. In autoregression, observations of $Y_{t}$ are regressed on previous observations $Y_{t-i}$. In other words, the process is regressed upon itself. $\operatorname{An} \operatorname{AR}(p)$ model of order $p$ takes the form:

$$
\boldsymbol{y}_{k}=\sum_{i=1}^{p} \operatorname{diag}\left(\boldsymbol{\alpha}_{i}\right) \boldsymbol{y}_{k-i}+\boldsymbol{\varepsilon}_{k}
$$

Where $\varepsilon_{k} \sim \mathcal{N}\left(0, \sigma^{2}\right)$.

A predictive model of control disturbance can be produced by solving a least-squares optimization problem. The objective is to minimize the Mean Squared Error (MSE) between $n$ prior disturbances $\boldsymbol{d}_{k-i}$ and predicted values $\widehat{\boldsymbol{d}}_{k-i}$ in the optimization process:

$$
\begin{gathered}
\text { Minimise: } \frac{1}{n-p} \sum_{i=1}^{n-p}\left(\boldsymbol{d}_{k-i}-\widehat{\boldsymbol{d}}_{k-i}\right)^{2} \\
\text { Subject to: } \widehat{\boldsymbol{d}}_{k-i}=\sum_{j=1}^{p} \operatorname{diag}\left(\boldsymbol{\alpha}_{j}\right) \boldsymbol{d}_{k-i-j}
\end{gathered}
$$

And $n \geq p+1$

Solving for $\boldsymbol{\alpha}$, the estimated control disturbance at time $k$ is then given by:

$$
\widehat{\boldsymbol{d}}_{k}=\sum_{i=1}^{p} \operatorname{diag}\left(\boldsymbol{\alpha}_{i}\right) \boldsymbol{d}_{k-i}
$$

Predictions at $k+N$ steps in the future can be made by substituting predicted values at time $k, k+1, \ldots, k+N-1$ in to the $\operatorname{AR}(p)$ model.

\section{B. Advantages to Autoregression}

Previous attempts at modelling wave disturbance have considered Gaussian, linear processes [6], and superposition of sinusoids [7] to name a few. However, AR holds a few advantages for this application. It is a simple linear model that, in the case of vehicle body motion, requires minimal data points $(n<20)$ in order to produce accurate forecasts. And by incorporating manipulator joints in to the state and control space (8), it is theoretically possible to predict and control for disturbance to the manipulator.

\section{Determining values for $n, p$}

Accuracy of the AR model depends on $p$ - the order of the model/number of $\alpha$ coefficients, and the number of prior constraint equations $(n-p)$. For example, an AR(2) model requires a minimum of $n=p+1=3$ data points to produce a prediction using 1 constraint equation.

Values of $n$ from 10 to 15 were found to be the most accurate, depending on the value of $p$. Interestingly, values of $n>20$ decreased the accuracy of forecast models. This implies that only the most recent motion data is useful in producing forecasts due to the erratic and constantly changing water surface conditions.

Fig. 3 shows forecasts from 3 AR models compared to vehicle motion data. AR has been noted as being inaccurate over long term forecasts [6]. However, as indicated in Fig. 3, it is sufficiently accurate within a forecast horizon of less than 0.5 seconds. Predictions for surge, sway, and heave are also more accurate than roll, pitch, and yaw motion. This has also been noted in [7].

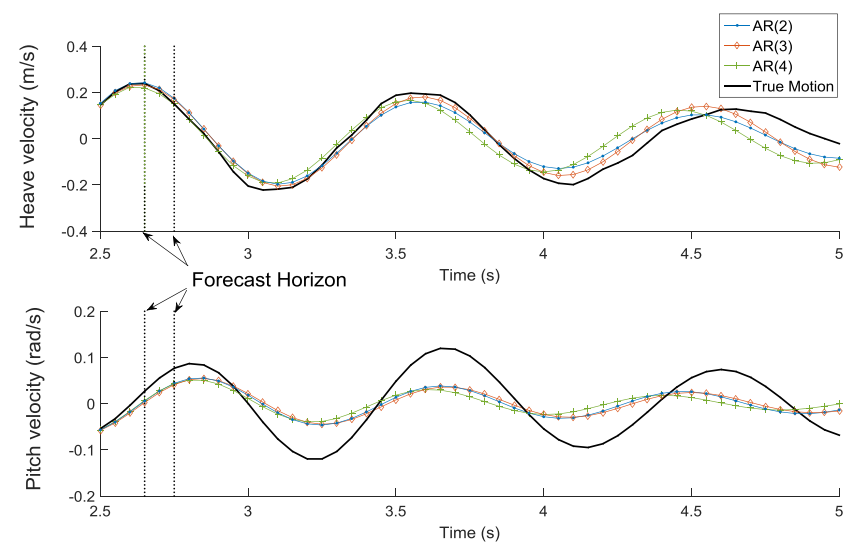

Figure 3. Forecasts from different orders of AR versus vehicle heave (top), and pitch (bottom) versus true motion

\section{MODEL PREDICTIVE CONTROL STRATEGY}

\section{A. Mathematical Modelling}

We consider a discrete time system of the form:

$$
\widehat{\boldsymbol{x}}_{k+1}=\boldsymbol{A} \boldsymbol{x}_{k}+\boldsymbol{B} \boldsymbol{u}_{k}+\boldsymbol{E} \widehat{\boldsymbol{d}}_{k}
$$

Where $\widehat{\boldsymbol{x}}_{k+1}$ is our expected state at the next time step based on predicted disturbance $\widehat{\boldsymbol{d}}_{k}$ from (3). form:

A general, discrete-time system is often written in the

$$
\begin{aligned}
\boldsymbol{x}_{k+1} & =\boldsymbol{A} \boldsymbol{x}_{k}+\boldsymbol{B} \boldsymbol{u}_{k}+\boldsymbol{E} \boldsymbol{w}_{k} \\
\boldsymbol{z}_{k} & =\boldsymbol{C}_{z} \boldsymbol{x}_{k}+\boldsymbol{D} \boldsymbol{u}_{k}
\end{aligned}
$$

In which $\boldsymbol{w}_{k}$ is assumed to be zero-mean Gaussian white noise. From (2) and (3) we can see that this parameter has been subsumed in to $\widehat{\boldsymbol{d}}_{k}$ of (4) and hence this convention is implicitly retained. If $\boldsymbol{B}_{k}=\boldsymbol{E}_{k}$, this can be abbreviated to:

$$
\begin{aligned}
\widehat{\boldsymbol{x}}_{k+1} & =\boldsymbol{A} \boldsymbol{x}_{k}+\boldsymbol{B}\left(\boldsymbol{u}_{k}+\widehat{\boldsymbol{d}}_{k}\right) \\
\boldsymbol{z}_{k} & =\boldsymbol{C} \boldsymbol{x}_{k}+\boldsymbol{D}\left(\boldsymbol{u}_{k}+\boldsymbol{d}_{k}\right)
\end{aligned}
$$

Where $\boldsymbol{z}_{k}$ is our observation of the true state, and $\boldsymbol{d}_{k}$ is the true, as-of-yet unknown control disturbance at the previous time step. Assuming that $\boldsymbol{A}=\boldsymbol{C}, \boldsymbol{B}=\boldsymbol{D}$, and that $\mathbf{B}$ is square and invertible, the previous control disturbance can be determined by:

$$
\boldsymbol{d}_{k}=\boldsymbol{B}_{k}^{-1}\left(\boldsymbol{z}_{k}-\boldsymbol{x}_{k+1}\right)+\widehat{\boldsymbol{d}}_{k}
$$




\section{B. Development of the system model}

We can define our state vector as:

$$
\boldsymbol{x}=\left[\begin{array}{c}
\boldsymbol{q} \\
\boldsymbol{\eta}_{e e}
\end{array}\right], \dot{\boldsymbol{x}}=\left[\begin{array}{c}
\dot{\boldsymbol{q}} \\
\dot{\boldsymbol{\eta}}_{e e}
\end{array}\right]
$$

Then from (1)

$$
\dot{\boldsymbol{x}}=\left[\begin{array}{cc}
\boldsymbol{I}_{6} & \mathbf{0}_{6 \times 6} \\
\boldsymbol{J}_{e, q}^{-1}\left(\boldsymbol{R}_{I}^{E}\right) \boldsymbol{J}(\boldsymbol{q}) & \boldsymbol{M} \boldsymbol{J}_{e, b}^{-1}\left(\boldsymbol{R}_{I}^{B}\right)
\end{array}\right]\left[\begin{array}{l}
\dot{\boldsymbol{q}} \\
\boldsymbol{v}
\end{array}\right]
$$

Then following the form of (4)

$$
\left[\begin{array}{c}
\boldsymbol{q} \\
\boldsymbol{\eta}_{e e}
\end{array}\right]_{k+1}=\left[\begin{array}{c}
\boldsymbol{q} \\
\boldsymbol{\eta}_{e e}
\end{array}\right]_{k}+\Delta T\left[\begin{array}{cc}
\boldsymbol{I}_{6} & \mathbf{0}_{6 \times 6} \\
\boldsymbol{J}_{e, q}^{-1}\left(\boldsymbol{R}_{I}^{E}\right) \boldsymbol{J}(\boldsymbol{q}) & \boldsymbol{M J}_{e, b}^{-1}\left(\boldsymbol{R}_{I}^{B}\right)
\end{array}\right]_{k}\left[\begin{array}{c}
\dot{\boldsymbol{q}}+\dot{\boldsymbol{q}}_{n} \\
\boldsymbol{v}+\boldsymbol{v}_{n}
\end{array}\right]_{k}
$$

Where $\boldsymbol{A}=1, \boldsymbol{B}=\Delta T\left[\begin{array}{cc}\boldsymbol{I}_{6} & \mathbf{0}_{6 \times 6} \\ \boldsymbol{J}_{e, q}^{-1}\left(\boldsymbol{R}_{I}^{E}\right) \boldsymbol{J}(\boldsymbol{q}) & \boldsymbol{M J}_{e, b}^{-1}\left(\boldsymbol{R}_{I}^{B}\right)\end{array}\right], \Delta T$ is the discrete time step, $\boldsymbol{u}=\left[\begin{array}{c}\dot{\boldsymbol{q}} \\ \boldsymbol{v}\end{array}\right]$, and $\boldsymbol{d}=\left[\begin{array}{c}\dot{\boldsymbol{q}}_{n} \\ \boldsymbol{v}_{n}\end{array}\right]$

\section{Model Predictive Control}

We wish to control the state of the end effector $\boldsymbol{\eta}_{e e}$ to some desired state $\boldsymbol{\eta}_{e e, d}$, with consideration for the effect that future disturbances will have on achieving this goal. Model Predictive Control (MPC) is pragmatic for this situation because of its ability to optimize the control plan for $\mathrm{N}$ future predictions. By factoring in forecasts of vehicle body motion, the model can devise the optimal control strategy to counter these disturbances, whilst adhering to any costs and strict constraints.

We first measure the current end-effector pose at the current time $\boldsymbol{\eta}_{e e, 0}$, and solve the MPC problem to return the optimal control $\boldsymbol{u}^{*}=\boldsymbol{u}_{0}$ :

$$
\text { minimise } J_{N}\left(\boldsymbol{\eta}_{e e, 0}, \boldsymbol{u}_{k}\right):=\sum_{k=0}^{N-1} \ell\left(\boldsymbol{\eta}_{e e, k}\left(\boldsymbol{\eta}_{e e, 0}\right), \boldsymbol{u}_{k}\right)
$$

Subject to:

$$
\begin{gathered}
\boldsymbol{\eta}_{e e, k+1}\left(\boldsymbol{\eta}_{e e, 0}\right)=f\left(\boldsymbol{\eta}_{e e, k}\left(\boldsymbol{\eta}_{e e, 0}\right), \boldsymbol{u}_{k}\right) \\
\left|\dot{\boldsymbol{q}}_{i, k}\right| \leq \dot{\boldsymbol{q}}_{i, \max } \\
\boldsymbol{q}_{i, L O W} \leq \boldsymbol{q}_{i, k+1} \leq \boldsymbol{q}_{i, U P} \\
\boldsymbol{v}_{k}=0
\end{gathered}
$$

Within this optimization problem, the AUVMS body velocity control $\boldsymbol{v}_{k}$ is set to zero. Waves at the surface of the water may be frequent and erratic. Furthermore, water flow around piles is turbulent and highly irregular [15]. Due to the time delay in the dynamic response of the vehicle control, we assume that it will be insufficient in nullifying these disturbances completely. Consequently, vehicle stability is solved as a separate problem. This also reduces complexity of the aforementioned optimization problem. In either scenario, any motion of the body is viewed as disturbance by the control method; hence we constrain the joint manipulators to counter its effect.

The cost function $\ell$ consists of 3 terms. The first term is the sum of the distance squared between the desired position and the expected position at the next time step (as a function of the predicted disturbance):

$$
\ell_{\text {distance }}=\left\|\boldsymbol{\eta}_{e e 1, d}-\widehat{\boldsymbol{\eta}}_{e e 1, k+1}\right\|^{2}
$$

The second term represents a cost for the error between the expected orientation of the end effector and the desired orientation:

$$
\ell_{\text {attitude }}=(\Delta \theta)^{2}
$$

In which the angle error $\Delta \theta$ is given by:

$$
\Delta \theta=\operatorname{acos}\left(\frac{\operatorname{trace}\left(\widehat{\boldsymbol{R}}_{k+1} \boldsymbol{R}_{d}^{T}\right)-1}{2}\right)
$$

Where $\boldsymbol{R}$ is a rotation matrix as a function of the end-effector attitude $\boldsymbol{\eta}_{e e 2}$.

Lastly, we consider $\ell_{\text {acceleration }}$ as the sum of the squared accelerations of the manipulator joints, which ensure smooth motion of the manipulator and end-effector.

$$
\ell_{\text {acceleration }}=\ddot{\boldsymbol{q}}_{k}^{T} \ddot{\boldsymbol{q}}_{k}
$$

Where the acceleration is given by:

$$
\ddot{\boldsymbol{q}}_{k}=\Delta T^{-1}\left(\dot{\boldsymbol{q}}_{k}-\dot{\boldsymbol{q}}_{k-1}\right)
$$

Finally, the total cost function is expressed as:

$$
\ell\left(\boldsymbol{\eta}_{e e, k}\left(\boldsymbol{\eta}_{e e, 0}\right), \boldsymbol{u}_{k}\right)=\left[\begin{array}{lll}
w_{1} & w_{2} & w_{3}
\end{array}\right]\left[\begin{array}{c}
\ell_{\text {distance }} \\
\ell_{\text {attitude }} \\
\ell_{\text {acceleration }}
\end{array}\right]
$$

Cost function weightings $w_{i}$ were chosen heuristically. Generally, $w_{2} \gg w_{1}, w_{3}$ to avoid situations where the optimization will trade off large attitude error to reduce position error. Values of $\left[\begin{array}{llll}w_{1} & w_{2} & w_{3}\end{array}\right]=$ [1 200 1] appeared to be sufficient.

\section{V.PROCESS OF THE END-EFFECTOR POSITION CONTROL}

A general process of the end effector position control is given in Fig. 4. A desired state is first defined somewhere within reach of the manipulator. If there is a difference between the desired state and the current known state, then the joint velocities are optimized to achieve it with consideration for any predicted disturbances.

After moving, the current state is then measured against the expected state. From this, prior disturbances can be determined. This is then added to a finite history of data. Old disturbance data is discarded as more recent information is acquired. Because of this moving window of prior disturbance, the AR model is constantly updated. This gives it the ability to adapt to changing external conditions. 


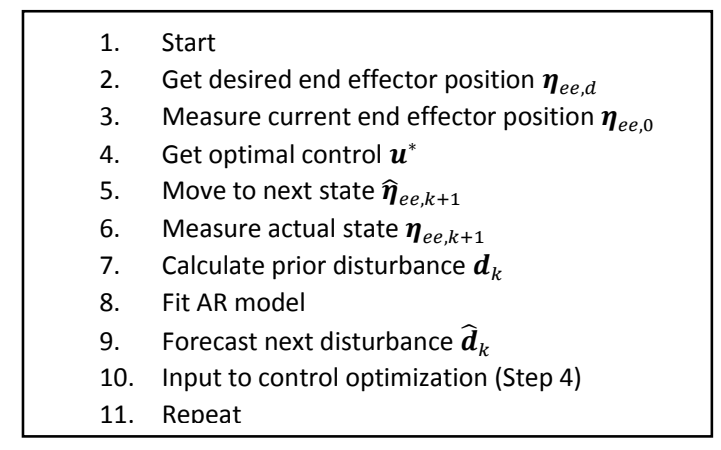

Figure 4. Process for kinematic control with AR and MPC

\section{SIMULATION AND RESULTS}

\section{A. Scenario and assumptions}

Real data was collected from an IMU sitting on a small vessel floating in a large body of water. Waves were generated to create a disturbance. This data was then fed through the simulation as $\boldsymbol{v}_{n}$ in (9). The kinematic model was simulated in MATLAB. As such, it is difficult to estimate the computational cost for real-time applications. Future work will involve conversion of the algorithm in to $\mathrm{C}++$ and running it on limited hardware to verify its feasibility.

A few assumptions were made when transferring the data to the simulation. Firstly, we assume that the AUVMS can maintain adequate heading towards the desired target. Ergo, large yaw velocities were scaled down. It is also assumed that the average position of the body over time does not move from its initial state i.e. velocities have a mean of 0 . It is also assumed that there is full knowledge of the AUVMS body pose in the inertial frame at any given time.

An arbitrary location was specified in the body frame $\{B\}$ at time $\mathrm{t}=0$. An arbitrary pose within reach of the manipulator was then defined as the goal in the task space. Using the control algorithm, the simulated AUVMS is expected to maintain this end-effector pose indefinitely. The simulation was run for 5 seconds, with sampling and forecasting frequencies of $20 \mathrm{~Hz}$. Manipulator joint velocities were capped at $1.2 \mathrm{rad} / \mathrm{s}$.

\section{B. Results of the simulation}

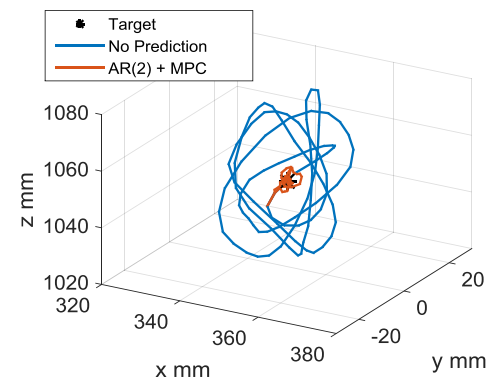

Figure 5. End-effector position over time: using no prediction and control (blue), versus $\mathrm{AR}(2)$ and MPC with $\mathrm{N}=3$ (red).
Fig. 5 shows the position of the end-effector in the inertial frame throughout the simulation. As a baseline, no prediction method and no model predictive control was used. In this manner, the manipulator simply tries to correct for error from the target after deviation has already occurred. In comparison, $\mathrm{AR}(2)$ prediction and MPC with a prediction horizon of $\mathrm{N}=3$ shows greatly improved accuracy. Simulations show a reduction in mean position error of $88 \%$ (Table 2). Furthermore, the variance was reduced by over half. This means the AR prediction model improves accuracy as well as certainty of the end-effector position at any given time.

It was noted by Becker et al [12] that AR models can reduced unwanted tremor in tele-operated surgical robots by $47 \%$ compared to a last-value predictor of disturbance. These simulations confirm a similar efficacy of AR over last-value predictions, in which an $\mathrm{AR}(2)$ model reduces error by approximately $65 \%$ using MPC.

The distance of the end-effector from the target, and the angle-error from the desired orientation was plotted over time for 3 types of predictive models: no prediction, last-value, and AR(2) (Fig. 6). The latter two were implemented with MPC. Despite inaccuracies in forecasts of pitch angles (Fig. 3 ), with similar observations in [7], it was found that AR still provides a much better reduction in rotation error than a lastvalue predictor.

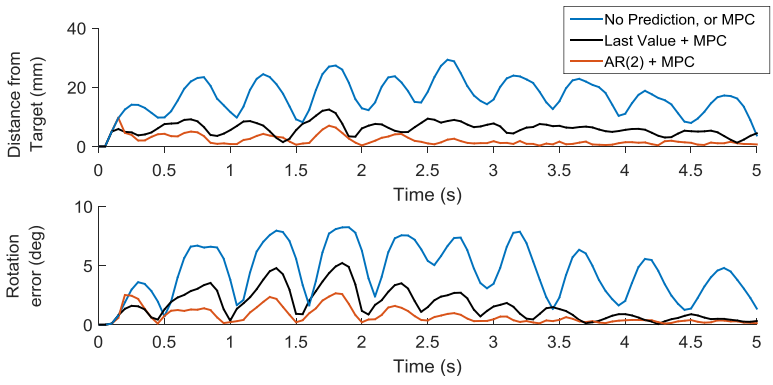

Figure 6. (Top) Distance of the end-effector from target and (bottom) angle error from the desired rotation.

It was also found that amongst all the AR models that there are diminishing returns in accuracy for longer forecast horizons. A horizon of $\mathrm{N}=5$ improved accuracy by less than $0.1 \mathrm{~mm}$ than that of $\mathrm{N}=3$ across all orders of AR. In the case of AR(4), it was found that accuracy began to decrease as the prediction horizon increased. It can be seen in Fig. 3 that forecasts of $\mathrm{AR}(4)$ diverge further over time from the true data compared to lower order models. This is likely due to the additional coefficients in the $\mathrm{AR}(4)$ model generating higher-order polynomials in the forecasts, which deviate from the true motion over time.

\section{CONCLUSION AND FUTURE WORK}

In this paper, it was shown that an autoregressive model of AUVMS body motion in rough water can be used to produce predictions of future body motion. Simulations were run in which these predictions were integrated in to model 
predictive control of an AUVMS end-effector. In doing so, it was shown that the joints of a manipulator can be used to cancel out unwanted motion caused by the vehicle body, and thus keep the end effector relatively stationary in the task space. An AR(2) model was found to be the simplest model for the most accurate disturbance prediction, with greater efficacy than a last-value predictor.

The outcomes of this research lead to multiple avenues for continued verification and development. Firstly, there is the concern for computational costs when running online on the AUVMS. This algorithm will need to be implemented on a real system to assure feasible solution times and memory consumption.

Future model development will also involve more robust kinematic control. This will include trajectory tracking under similar conditions in order to carry out cleaning of a pile surface, and utilization of redundant kinematics for joint-limit and singularity avoidance. Dynamic effects may also need to be accounted for. However, the 6DOF manipulator is cable driven and predominantly made of plastic. Therefore, effects such as inertia and torque loads are likely to be minimal.

Finally, we intend to implement this model predictive control method on the AUVMS prototype. Experimental testing can then be conducted both in laboratory settings, and during on-site trials to substantiate its accuracy and robustness in different operating conditions.

\section{ACKNOWLEDGMENT}

The authors thank Dr. Stephen Woodcock for the initial insight in to prediction with ARMA models. We would also like to thank Brendan Emery and Luke Coffey for their assistance with data collection.

\section{APPENDIX}

TABLE 2. SIMULATION RESULTS FOR DIFFERENT PREDICTION MODELS AND DIFFERENT FORECAST HORIZONS OF MODEL PREDICTIVE CONTROL.

\begin{tabular}{cccccc}
$\mathbf{N}$ & $\begin{array}{c}\text { Prediction } \\
\text { Model }\end{array}$ & $\begin{array}{c}\text { Mean } \\
\text { Position } \\
\text { Error } \\
(\mathbf{m m})\end{array}$ & Std. Dev. & $\begin{array}{c}\text { Angle } \\
\text { Error } \\
(\mathbf{d e g})\end{array}$ & $\begin{array}{c}\text { Std. } \\
\text { Dev. }\end{array}$ \\
\hline \multirow{4}{*}{1} & None & 16.78 & 5.98 & 4.54 & 2.24 \\
& Last Value & 10.50 & 4.35 & 4.82 & 2.34 \\
& AR(2) & 9.46 & 4.06 & 4.58 & 2.24 \\
& AR(3) & 9.50 & 4.11 & 4.61 & 2.25 \\
& AR(4) & 9.44 & 4.01 & 4.56 & 2.24 \\
\hline \multirow{4}{*}{3} & None & 14.01 & 5.14 & 1.63 & 1.09 \\
& Last Value & 5.99 & 2.29 & 1.63 & 1.31 \\
& AR(2) & 2.07 & 1.68 & 0.73 & 0.67 \\
& AR(3) & 2.07 & 1.64 & 0.74 & 0.68 \\
& AR(4) & 2.23 & 1.65 & 0.69 & 0.64 \\
\hline \multirow{2}{*}{5} & None & 14.07 & 5.13 & 1.80 & 1.14 \\
& Last Value & 5.86 & 2.21 & 1.57 & 1.24 \\
& AR(2) & 1.98 & 1.36 & 0.69 & 0.50 \\
& AR(3) & 2.00 & 1.31 & 0.72 & 0.55 \\
& AR(4) & 2.36 & 2.05 & 0.77 & 0.56
\end{tabular}

Notation for the AUVMS kinematic equations:

- I, B, O, E: Inertial, Body, Base, and End-Effector frames respectively.

- $\boldsymbol{\eta}_{e e}=\left[\begin{array}{l}\boldsymbol{\eta}_{e e 1} \\ \boldsymbol{\eta}_{e e 2}\end{array}\right]=\left[\begin{array}{ccc}{\left[\begin{array}{lll}x & y & z\end{array}\right]^{T}} \\ {\left[\begin{array}{lll}\phi & \theta & \varphi\end{array}\right]^{T}}\end{array}\right]$ : End effector pose in I.
- $\quad \boldsymbol{\eta}=\left[\begin{array}{l}\boldsymbol{\eta}_{1} \\ \boldsymbol{\eta}_{2}\end{array}\right]$ : Vehicle pose in I

- $\quad \boldsymbol{R}_{I}^{B}$ : Rotation matrix from I to B

- $\boldsymbol{R}_{I}^{O}$ : Rotation matrix from I to O

- $\overrightarrow{\boldsymbol{r}}_{b o}$ : Displacement from B to O with respect to B.

- $\overrightarrow{\boldsymbol{r}}_{o e}$ : Displacement from $\mathrm{O}$ to $\mathrm{E}$ with respect to $\mathrm{O}$

- $\boldsymbol{J}_{k o}\left(\boldsymbol{\eta}_{2}\right)$ : Angular velocity transformation

\section{REFERENCES}

[1] Cieslak, P. et al "Autonomous Underwater Panel Operation by GIRONA500 UVMS: A Practical Aproach to Autonomous Underwater Manipulation", May 26-30 2015 IEEE International Conference on Robotics and Automation, pp. 529 - 536

[2] Simetti et al, "Floating Underwater Manipulation: Developed Control Methodology and Experimental Validation within the TRIDENT Project" 2013, Journal of Field Robotics 31, pp. $364-385$

[3] Yuh et al, "Design of a Semi-Autonomous Underwater Vehicle for Intervention Missions (SAUVIM)", Proceedings of the 1998 International Symposium on Underwater Technology, pp. $63-68$

[4] Marani, G., Yuh, J. "Introduction to Autonomous Manipulation: Case Study with an Underwater Robot, SAUVIM" (C) Springer-Verlag Berlin Heidelberg 2014

[5] Marani, G. et al "Underwater autonomous manipulation for intervention missions AUVs" 2009, Ocean Engineering 36, pp. $15-$ 23

[6] Yang et al, "Ship Motion Prediction for Maritime Flight Operations", 2008, Proceedings of the $17^{\text {th }}$ World Congress, The International Federation of Automatic Control, pp. 12407 - 12412

[7] From et al, "Motion planning and control of robotic manipulation on seaborne platforms", 2011, Control Engineering Practice, Vol.9 Issue 8, pp. 809-819

[8] Huynh, V. et al, "Predictive Motion Planning for AUVs subject to strong time-varying currents and forecasting uncertainties" Proceedings of the IEEE International Conference on Robotics and Automation, May 2015, pp.1144 - 1151

[9] Galceran et al, "Coverage Path Planning with Realtime Replanning for Inspection of Underwater Structures", 2014 IEEE International Conference on Robotics \& Automation (ICRA), pp. 6586 - 6591

[10] Ohshima et al, "Model predictive control with adaptive disturbance prediction and its applications to fatty acid distillation column control”, Journal of Process Control, Vol. 5, No. 1 ,January 1995, pp. $41-48$

[11] Oldewurtel et al, "Use of model predictive control and weather forecasts for energy efficient building climate control", Energy and Buildings 45, 2012, pp. 15-27

[12] Becker, B. et al "Autoregressive Modeling of Physiological Tremor under Microsurgical Conditions" Conf Proc IEEE Eng Med Biol Soc. 2008, pp. $1948-1951$

[13] Southgate, T. Myers, A., "Mussel Fouling on the Celtic Sea Kinsale Field Gas Platforms", 1985, Estuarine, Coastal and Shelf Science vol. 20 , pp. $651-659$

[14] Kerckhof et al, "Early colonisation of a concrete offshore windmill foundation by marine biofouling on the Thornton Bank southern North Sea): first monitoring results", 2009, Underwater Technol.: Int. J. Soc. Underwater 29 pp. $137-149$

[15] Keshavarzi, A., Melville, B, Ball, J. "Three-dimensional analysis of coherent turbulent flow structure around a single circular bridge pier", 2014 Environmental Fluid Mechanics vol. 14 pp. 821 - 847

[16] Antonelli, G. "Underwater Robots" 2006, @ Springer-Verlag Berlin Heidelberg 